\title{
BMJ Open Multidimensional dynamic healthcare personnel (HCP)-centric model from a low-income and middle-income country to support and protect COVID-19 warriors: a large prospective cohort study
}

To cite: Dutta U, Sachan A, Premkumar M, et al. Multidimensional dynamic healthcare personnel (HCP)-centric model from a low-income and middleincome country to support and protect COVID-19 warriors: a large prospective cohort study. BMJ Open 2021;11:e043837. doi:10.1136/ bmjopen-2020-043837

- Prepublication history and supplemental material for this paper is available online. To view these files, please visit the journal online (http://dx.doi. org/10.1136/bmjopen-2020043837).

Received 17 August 2020 Revised 11 November 2020 Accepted 17 November 2020

Check for updates

(c) Author(s) (or their employer(s)) 2021. Re-use permitted under CC BY-NC. No commercial re-use. See rights and permissions. Published by BMJ.

For numbered affiliations see end of article.

Correspondence to

Dr Usha Dutta;

ushadutta@gmail.com

Usha Dutta, ${ }^{1}$ Anurag Sachan, ${ }^{1}$ Madhumita Premkumar (D) , ${ }^{1,2}$ Tulika Gupta, ${ }^{3}$ Swapnajeet Sahoo, ${ }^{4}$ Sandeep Grover, ${ }^{4}$ Sugandhi Sharma (i) , ${ }^{5}$ P V M Lakshmi, ${ }^{5}$ Shweta Talati, ${ }^{6}$ Manisha Biswal, ${ }^{7}$ Vikas Suri, ${ }^{8}$ Mini P Singh, ${ }^{9}$ Babita Ghai, ${ }^{10}$ Rajesh Chhabra, ${ }^{11}$ Bhavneet Bharti, ${ }^{12}$ Jayanta Samanta, ${ }^{1}$ Pankaj Arora, ${ }^{6,8}$ Ritin Mohindra, ${ }^{8}$ Sunita Malhotra, ${ }^{13}$ Gurmeet Singh, ${ }^{5}$ Rashmi Ranjan Guru, ${ }^{6}$ Navin Pandey, ${ }^{6}$ Vipin Koushal, ${ }^{6}$ Ashok Kumar, ${ }^{6}$ Ranjitpal Singh Bhogal, ${ }^{6}$ Arun K Aggarwal, ${ }^{5}$ Kapil Goel, ${ }^{5}$ Pankaj Malhotra, ${ }^{8}$ Narayana Yaddanapudi, ${ }^{10}$ Pranay Mahajan, ${ }^{6} \mathrm{~J} S$ Thakur (1) , ${ }^{5}$ Rakesh Sehgal, ${ }^{14}$ Arnab Ghosh, ${ }^{9}$ Inderpaul Singh Sehgal (D) , ${ }^{15}$ Ritesh Agarwal, ${ }^{15}$ Muralidharan Jayashree, ${ }^{12}$ Ashish Bhalla, ${ }^{8}$ Sanjay Jain,, ${ }^{8}$ Rakesh Kochhar (D) , ${ }^{1}$ Arunaloke Chakrabarti, ${ }^{7}$ Goverdhan Dutt Puri, ${ }^{10}$ Jagat Ram ${ }^{16}$

\section{ABSTRACT}

Objectives Healthcare personnel (HCP) are at an increased risk of acquiring COVID-19 infection especially in resourcerestricted healthcare settings, and return to homes unfit for self-isolation, making them apprehensive about COVID-19 duty and transmission risk to their families. We aimed at implementing a novel multidimensional HCP-centric evidence-based, dynamic policy with the objectives to reduce risk of HCP infection, ensure welfare and safety of the HCP and to improve willingness to accept and return to duty. Setting Our tertiary care university hospital, with 12600 HCP, was divided into high-risk, medium-risk and low-risk zones. In the high-risk and medium-risk zones, we organised training, logistic support, postduty HCP welfare and collected feedback, and sent them home after they tested negative for COVID-19. We supervised use of appropriate personal protective equipment (PPE) and kept communication paperless.

Participants We recruited willing low-risk HCP, aged $<50$ years, with no comorbidities to work in COVID-19 zones. Social distancing, hand hygiene and universal masking were advocated in the low-risk zone.

Results Between 31 March and 20 July 2020, we clinically screened 5553 outpatients, of whom 3012 (54.2\%) were COVID-19 suspects managed in the medium-risk zone. Among them, 346 (11.4\%) tested COVID-19 positive (57.2\% male) and were managed in the high-risk zone with 19 (5.4\%) deaths. One $(0.08 \%)$ of the $1224 \mathrm{HCP}$ in high-risk zone, $6(0.62 \%)$ of $960 \mathrm{HCP}$ in medium-risk zone and 23 $(0.18 \%)$ of the $12600 \mathrm{HCP}$ in the low-risk zone tested
Strengths and limitations of this study

- This is a large prospective cohort study using a dynamic healthcare personnel (HCP)-centric COVID-19 model from a low-income andmiddle-income country with high patient load and resource limitations.

- To reduce COVID-19 transmission, the hospital was divided into three risk zones, prolonged duty hours were avoided and HCP were provided training, personal protective equipment (PPE), boarding, lodging and transport during their duty period, and sent home only after they tested negative for COVID-19.

- We empowered HCP by establishing low-cost paperless communication system with their pre-existing mobile devices to efficiently access welfare services as well as provide prompt feedback.

- One major limitation of our study is lack of structured feedback from all the HCP.

- Other limitations are need for lead time of 2 weeks to set up the system, pre-existing infrastructure, skilled manpower, access to good quality PPE and low-cost internet facility which are necessary to adopt the model.

positive at the end of shift. All the 30 COVID-19-positive HCP have since recovered. This HCP-centric policy resulted in low transmission rates $(<1 \%)$, ensured satisfaction with training (92\%), PPE (90.8\%), medical and psychosocial support (79\%) 
and improved acceptance of COVID-19 duty with $54.7 \%$ volunteering for re-deployment.

Conclusion A multidimensional HCP-centric policy was effective in ensuring safety, satisfaction and welfare of HCP in a resource-poor setting and resulted in a willing workforce to fight the pandemic.

\section{BACKGROUND}

COVID-19 disease was declared a pandemic by WHO on 11 March 2020 and the first case in the city of Chandigarh, India was reported on 18 March 2020. The global COVID-19 pandemic has presented an enormous challenge to the existing healthcare system in different countries, causing a paradigm shift in healthcare practices. ${ }^{1-3}$ As of 17 October 2020, 39023292 confirmed cases of COVID-19 have been documented worldwide with 1099586 deaths in 216 countries. WHO estimates there are 13.8 million COVID-19 infections worldwide and that healthcare personnel (HCP) represent 1.4 million cases, about $10 \%$ of all COVID-19 cases. ${ }^{4}$ In India, there are 7 370468 documented infections until 17 October 2020 with 112998 deaths. ${ }^{5}$

HCP are at risk of contracting infection and transmitting it to their family and community. An estimated 2238 doctors have been infected in India with deaths of 382 doctors reported until 16 September $2020 .^{67}$ The rising COVID-19 cases in the country and increasing numbers of reported deaths among HCP raised an alarm among our Indian HCP. Apart from an increased risk of infection, the HCP also faced social stigma as they are considered potential sources of disease transmission in the local community. ${ }^{8}$ Many communities asked medical workers to leave rented lodging without notice or restricted movement in shops or the locality. This has resulted in apprehension and reluctance in their accepting duty as frontline workers as they feared repercussions for themselves and their family. ${ }^{9}$

Our institute, the Postgraduate Institute of Medical Education and Research (PGIMER), Chandigarh, India, was designated as a COVID-19 treatment centre on 21 March 2020 after the first case in the city was reported on 18 March 2020. Thus, an institutional multidisciplinary team (MDT) was created on 31 March 2020, which aimed at providing stewardship, coordination and policy specific to the institution, and created a web portal. ${ }^{10}$ We searched relevant literature, consulted relevant stakeholders to understand HCP concerns in our hospital, took resource constraints and feasibility issues into our consideration before drafting our policy. ${ }^{11}{ }^{12}$ We drafted policies and standard operating protocols (SOP), which were in consonance with the guidelines formulated by the Indian Council of Medical Research (ICMR), the Ministry of Health and Family Welfare, Government of India, WHO, the Centers for Disease Control and Prevention (CDC) and published global experience. ${ }^{13-19}$ A recent report highlighted access to personal protective equipment (PPE) was a key concern of HCP when handling suspected COVID-19 cases. Also, there were concerns of personal safety, anxiety related to transmitting the infection to their family, logistic issues related to home or work due to the nationwide lockdown. ${ }^{20}$ Healthcare systems are already overburdened with over $100 \%$ occupancy, and HCP should be protected and cared for as individuals by providing PPE, housing and transportation, training opportunities, allowances and timely salaries and public service awards. ${ }^{12}$

We aimed to gauge the success for our MDT model of COVID-19 care and HCP welfare by assessing the HCP preparedness, training adequacy, welfare satisfaction and HCP infection rates at different levels of care. Most published literature on COVID-19 disease is centred on patient care, transmission and clinical outcomes. There is limited literature on the efforts of any centre in management of HCP welfare and safety. ${ }^{21-23}$ We report our realworld experience from a low-income and middle-income country with formulating a multidimensional HCP care policy, to train, prevent transmission and ensure overall welfare of our HCP while they continued to take care of the patients infected with COVID-19.

\section{METHODS}

The aim of the COVID-19 HCP welfare team was to understand the concerns of the HCP, to formulate HCPcentric policies, ensure logistic support for frontline HCP, to communicate their concerns to institute's administration and facilitate translating policies into action. To further these goals, a core committee for HCP welfare expanded to form the MDT to provide multidimensional HCP support. The 'HCP-centric' COVID-19 care policy was brought forth to complement the 'patient-centric' COVID-19 management policy of the institute..$^{10} 12$ 17-19 The objectives were to reduce risk of HCP infection, and ensure welfare and safety of the HCP, and to improve willingness to accept and return to duty.

\section{Multidisciplinary team}

The MDT was formed to train, protect and watch over the welfare of the HCP while ensuring optimum COVID-19 patient care. The team involved a rolling cohort of faculty and staff with experience in varying aspects of healthcare. The core team was headed by the director of the institute, and the senior faculty from internal medicine and subspecialities, anaesthesia and critical care, community medicine, microbiology, virology and hospital administration. Overall, it had 25 members as core team which supported the rolling cohort of HCP. This MDT comprised several co-dependent units. The administrative unit managed resources and manpower (figure 1A). The COVID-19 patient care unit was given the responsibility of day-to-day management of patients and the COVID-19. Laboratory testing unit oversaw the virological diagnosis protocols and testing of all biological samples of patients with COVID-19 while ensuring safety of laboratory personnel. The COVID-19 infection control unit procured, rationalised and distributed protective gear according to the three-tiered risk stratification. The COVID-19 HCP training unit prepared 


\section{A}

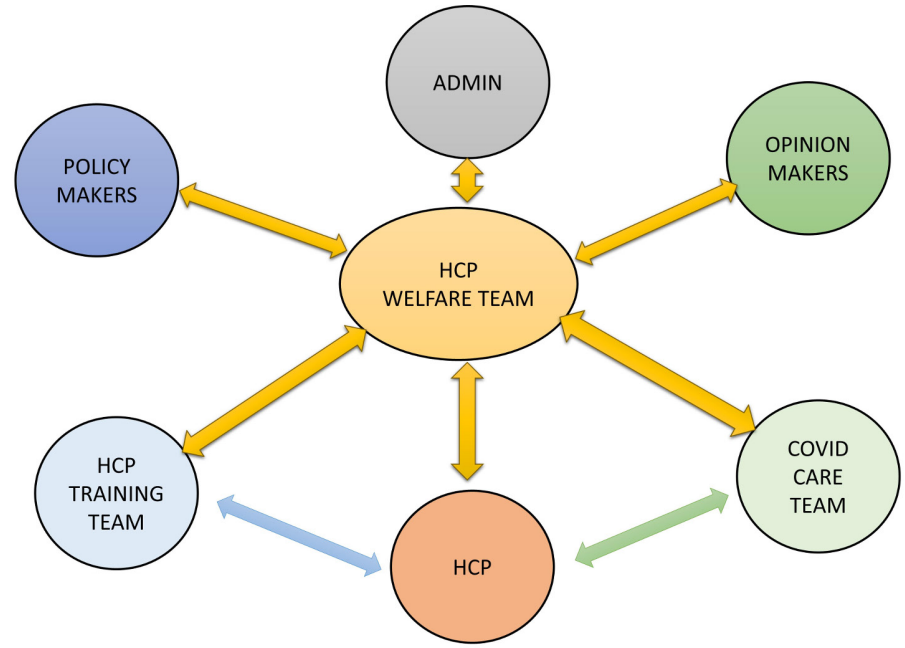

B

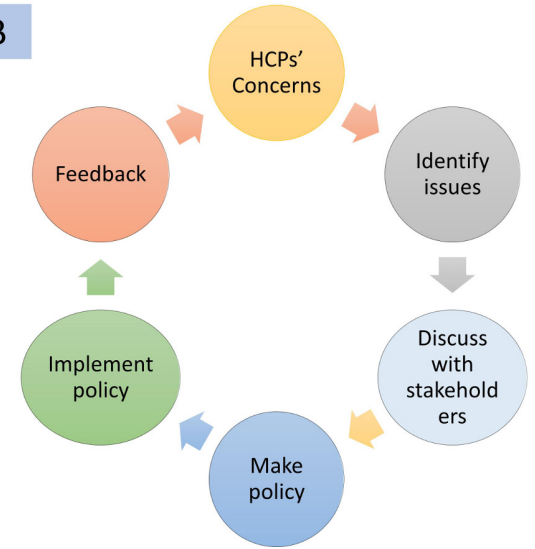

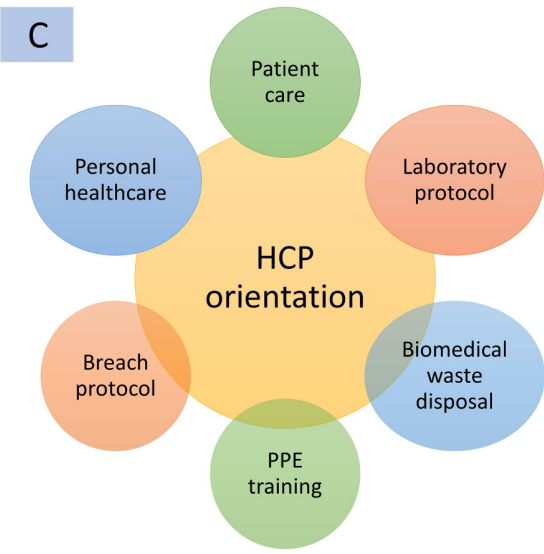

Figure 1 (A) Healthcare welfare team playing a pivotal role in coordinating collaborating and communicating with administrators, opinion makers and policy makers HCP training team and COVID-19 patient care team to provide a multidimensional HCP-centric care. (B) Finding an optimal solution of each of the HCP concerns and converting it into an HCP-centric policy. (C) Holistic training of the HCP before being deployed on COVID-19 duty regarding patient care, laboratory protocols, biomedical waste disposal, PPE training, breach protocols and personal healthcare. HCP, healthcare personnel; PPE, personal protective equipment.

the HCP for patient care, self- isolation, biomedical waste management and other aspects using an institute-specific curated manual and the COVID-19 HCP welfare unit was in charge of the health and safety, food and accommodation, transportation, medical needs of the deployed HCP and their postexposure COVID-19 testing. Although the core teams in each of units of the MDT were constant, nominated persons from various disciplines were added to supplement implementation when required (figure 1).

\section{Analysing the HCP concerns}

The HCP welfare team first analysed the existing issues and concerns faced at ground level by HCP by discussing with various stakeholders. ${ }^{18}$ The HCP deployed at COVID-19 care sites, their administrative supervisors and union representatives were consulted. We also involved the lodging in charges, transport personnel, food outlet providers, the biomedical waste management team in the decision-making process as secondary stakeholders. Each problem was assessed, and an optimal solution was proposed and executed (table 1). Most HCP in India, like many other low-income and middle-income nations, live in small shared living spaces among multigenerational households where they cannot self-isolate safely. Conversely, the HCP were also anxious about social media videos depicting difficult working environments and reports of deaths among healthcare workers. ${ }^{267}$ Some HCP suffered from anxiety, insomnia and depression due to the above concerns. ${ }^{24-26}$ Commuting to the hospital was another concern as public transport was not readily available due to country-wide lockdown. As most of our contractual HCP did not have any health insurance, we organised group insurance for them and provided token financial incentive to those who belonged to the lower socioeconomic class by the modified Kuppuswamy scale. ${ }^{27}$

\section{HCP-centric policy}

After assessing all the relevant HCP concerns at our centre, we reviewed relevant published data about experience in other countries and the ICMR and WHO guidelines to formulate our unique institute-specific policy. ${ }^{10-19}$ The HCP-centric COVID-19 care policy was devised using evidence-based principles (figure 2). We opted for a paperless, single window model, rapid response system 


\begin{tabular}{lll}
\hline Table 1 Concerns of healthcare workers and their addressal & \\
\hline Concern related to & Issues & Solution \\
\hline Self-related & Prevention of transmission & Good quality PPE and training in its use \\
& Isolation & Internet services \\
& Mental health & Medical team support \\
& Food & Mental health support \\
Family & Inability to self-isolate & Timely tasty and nutritious meals \\
& Crowded homes & Hospital provided accommodation \\
& High risk to family members & Sent home after negative testing \\
Transport & Lack of family & Group insurance \\
& Safe transport & Financial incentives to support staff \\
Hospital & Fear and apprehension & Hospital provided transport \\
& PPE & Drivers with PPE and use of sanitised vehicles \\
& Patient care & Preduty orientation and counselling \\
& Waste management & Training in donning and doffing \\
& Dehydration and hunger & SOPs and telemedicine support \\
& Heat in PPE & Biomedical waste management training \\
& Fear of ostracism by society & Preduty and postduty refreshments/fluids \\
& Fear of spreading in community & Ambient working temperature $\left(18^{\circ} \mathrm{C}-21^{\circ} \mathrm{C}\right)$
\end{tabular}

PPE, personal protective equipment; SOP, standard operating protocol.

to ensure efficient system, which also does not result in fomite transmission to HCP. This involved creating a webbased communication and data management system. The policy was dynamic and adapted as per the changing circumstances of the pandemic, availability of resources and feedback from other MDT units and the HCP (figure 3). We ensured practical measures like cancellation of non-essential activities, adopting web-based teaching, reducing outpatient services and switching to telemedicine, and prioritised human resources, providing rest, food, social and psychological support to all HCP.

\section{Definitions}

Healthcare personnel

WHO defines healthcare workers as 'all people engaged in actions whose primary intent is to enhance health'. For this protocol, we defined HCP as all employees of the hospital, whether on regular or contractual appointment, who were engaged in actions whose primary intent is to enhance health as per the WHO definition. ${ }^{28}$

\section{COVID-19 suspect cases}

A patient with acute respiratory illness with fever or at least one sign/symptom of respiratory disease, and a history of travel to an area or country with COVID-19 transmission OR a person or HCP with any respiratory illness AND a contact with a confirmed case OR a person with severe acute respiratory illness, OR a case in whom COVID-19 testing is inconclusive.

\section{COVID-19 confirmed cases}

A person with laboratory confirmation of COVID-19 infection, irrespective of clinical signs and symptoms. Further details are provided in the online supplemental file $1 .{ }^{1016}$

\section{Creation of a safe workspace for the HCP}

All patients underwent risk assessment in the screening areas, and then moved to the appropriate hospital unit as per perceived risk of COVID-19. The area where patients were screened and where COVID-19 suspects were retained for COVID-19 testing until reports were made available was designated as orange zone with medium risk of transmission. From the orange zone, on testing negative, they were moved to the general hospital services with low risk of transmission (green zone), and if positive they were moved to a separate building of the hospital, which was designated as the COVID-19 facility (red zone) (figure 3). This facility exclusively served confirmed COVID-19-positive cases as it was a high-risk zone for transmission. ${ }^{29}$ The building had five levels, and each of which was designated for a separate function. The first level was used for administration, and training of HCP, second, third and fourth levels were designated for stable patients requiring monitoring and the fifth level was designated for patients requiring intensive care. ${ }^{30}$ The staff was divided into two categories namely the 'supervisory team' who had no direct contact with patient care and the 'frontline team' who were directly managing the patients in duty shifts on a weekly roster. Separate work areas, movement corridors and lifts were designated for the on-duty staff and administration to minimise risk to the HCP. ${ }^{31}$

\section{Provision of HCP protective equipment and universal precautions}

All HCP received training regarding use of PPE specific to their area, hand hygiene, appropriate use of masks, disposal of waste and disinfection protocols. PPE was 


\section{HEALTHCARE PERSONNEL WELFARE POLICY}

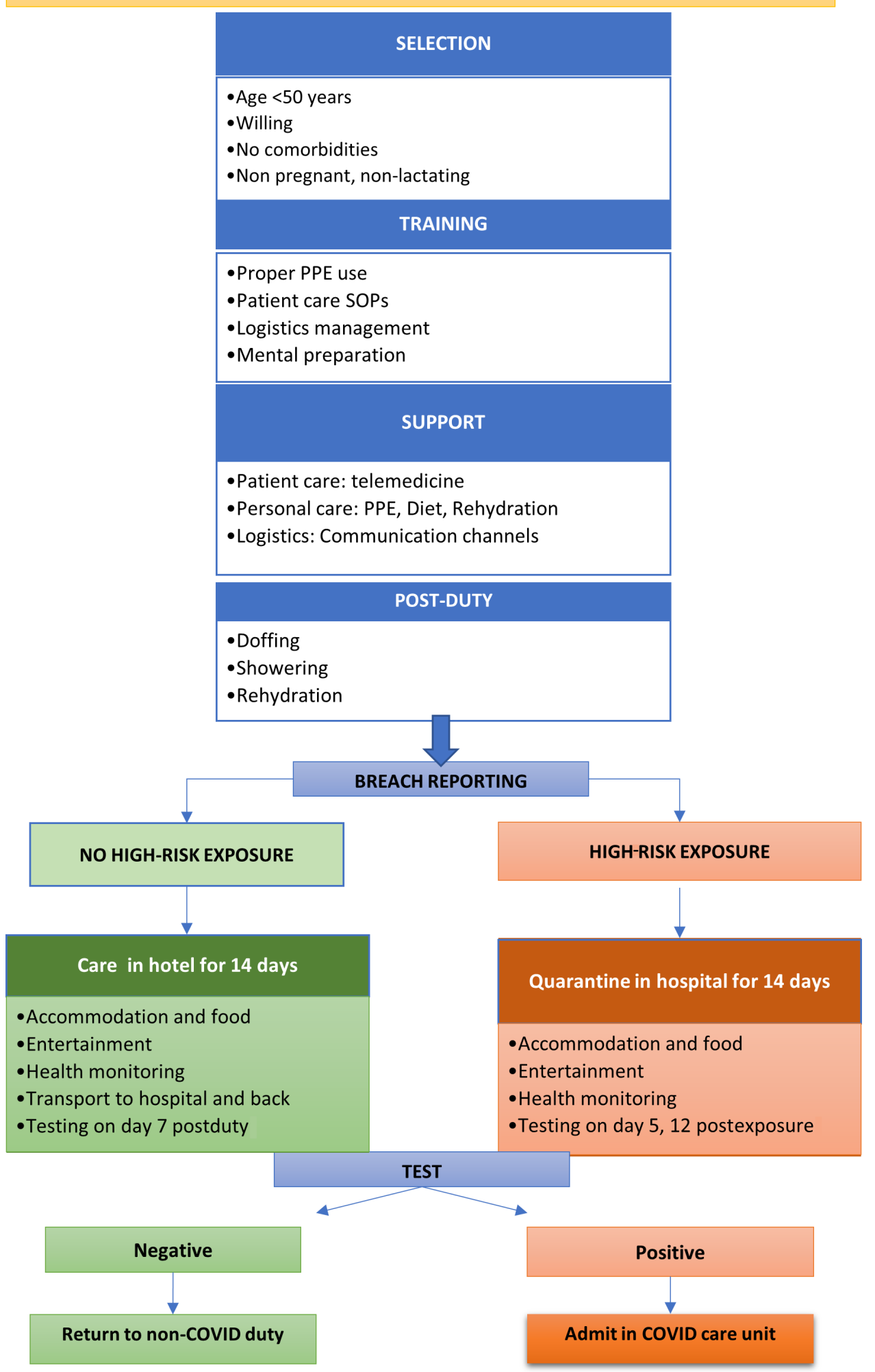

Figure 2 Standard operating policy for healthcare personnel recruitment, training, support, postduty isolation/quarantine and postduty COVID-19 testing. PPE, personal protective equipment; SOP, standard operating protocol.

provided as per the level of risk in the three zones. The red zone HCP had full PPE provision, with N95 respirators, coverall suits, visor, cap, gloves and dual shoe covers. The orange zone was provided triple layered surgical mask and surgical gown. In all areas with full PPE, the shift was limited to 6 hours, which avoided excessive workloads, gave time for rest and recreation. In case of any HCP becoming symptomatic or testing positive, 

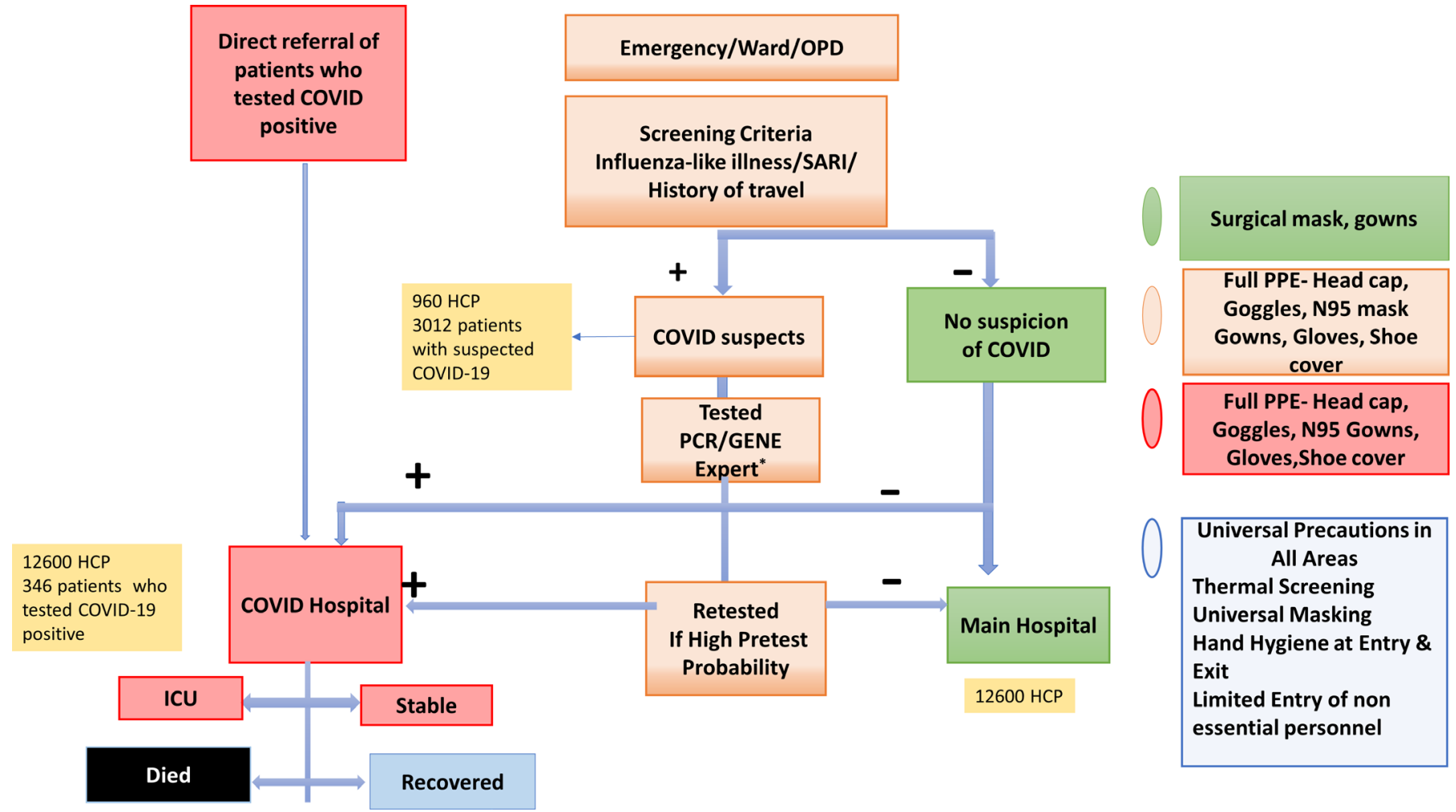

Figure 3 Three tiers of COVID-19 care zones - red (high-risk zone), orange (medium-risk zone) and green (low-risk zone) with the appropriate PPE in the respective areas to optimise and streamline resources. *PCR turnaround time (8-12 hours) -stable patents; GENE Expert turnaround time (2 hours) - critical patents. HCP, healthcare personnel; ICU, intensive care unit; ILI, influenza like illness; PPE, personal protective equipment; SARI, severe acute respiratory infection.

strict quarantine, contact tracing was done. The green zone HCP were provided triple layer surgical mask and asked to maintain strict social distancing and universal masking policy. ${ }^{23}$ Universal precautions in all three zones were universal use of masks, strict hand hygiene, thermal screening at entry, COVID-19 testing at patient admission points, restriction of admissions based on triage priority, restriction of non-essential visitors to the hospital and training of administration, security, sanitation, hostel and other personnel to reduce infections. The use of additional PPE like visors and intubation boxes was used in all areas for aerosol generating procedures. Recycling of N95 masks was advocated in hospital areas where hot air/ plasma sterilisation was possible, which reduced costs and provided additional support.

\section{Predeployment training of the HCP}

The eligibility criteria for frontline workers in the red and orange zones were HCP aged $<50$ years, non-pregnant, non-lactating women, without apparent cause for immunosuppression, those without comorbidities and those who were willing to perform patient care duty in the COVID-19 hospital as first responders. We prepared the HCP mentally for both the on-duty and postduty social isolation period by briefing them in a language understandable to them, about what to expect and how to handle issues (figure 1C). Training included pre-post duty instructions, accommodation information, use of PPE, sample collection and safe transportation to the laboratory, communication skills and information about emergency contact helplines. ${ }^{29}$ Specific hands on training for 'donning' and 'doffing' of PPE was done by video presentation and/or live demonstrations by faculty tutors. The remaining HCPs were provided training in the form of online videos and onsite visits with instructional material from the infection control team. The HCP were made aware of the frequently asked questions by the patients and their care givers, and they were taught how to manage patients without compromising their own safety. Those who had completed their tour of duty were drafted into the training exercise to provide practical inputs and motivate the new recruits.

\section{HCP support during the duty period}

Just before the duty, all HCP were provided with a high energy dry snack as use of diapers was not acceptable to most. Specified 'donning' rooms with mirrors with graphical step-by-step pictorial instructions were designed. ${ }^{30} 31 \mathrm{~A}$ few hand-signs were taught to all active workers for communicating via closed-circuit television (CCTV) cameras placed in the segregated donning and doffing room. A buddy ensured that they had appropriately donned and were comfortable in the PPEs before entering the 'duty' area. The duty area had a comfortable ambient temperature of $18^{\circ} \mathrm{C}-21^{\circ} \mathrm{C}$, controlled with an area-specific high-efficiency particulate air filtration handling unit. Communication channels were established between the HCP and the COVID-19 patient care 
unit using CCTV, computer and smartphones. 'Doffing' of PPE was identified as a crucial step and required patience and guidance for proper execution and thus it was specifically supervised by the infection control team by use of closed-circuit camera and assisted by a buddy if required. If anyone had a documented breach, they underwent a formal assessment to classify it as low-risk or a high-risk exposure. Those with high-risk exposure were counselled and quarantined in the private ward facility of our hospital. Those with low-risk exposure continued to work with self-monitoring and standard precautions.

\section{Peri-duty self-isolation facility}

A nearby hotel was exclusively dedicated to boarding and lodging facility for self-isolation of the HCP during the duty and postduty period. The hotel staff was equipped with appropriate PPE and trained in universal precautions and waste management. ${ }^{30}$ The interface between the HCP, the hotel and the HCP welfare unit were by digital interface with no face-to-face interaction to minimise fomite and person-to-person transmission. All the HCP were periodically screened telephonically for any medical and mental health issues. Anxiety and depression were assessed using questionnaire (Generalised Anxiety Disorder-7 and Patient Health Questionnaire-9), and if present they were counselled/provided appropriate medication. ${ }^{32}{ }^{33}$ Secure transportation facility to and from the hospital to the hotel was provided with sanitised vehicles and PPE-clad drivers.

The PPE-clad trained housekeeping staff disinfected the vacated rooms using a $1 \%$ hypochlorite spray and bed linen was disinfected before sending it for laundry.

\section{Postexposure COVID-19 testing of the HCP}

We routinely tested all HCP after their duty for presence of COVID-19 virus by checking their nasopharyngeal swab by COVID-19 PCR using TaqPath COVID-19 Combo kit (Thermo Fisher Scientific, USA). It has a reported sensitivity of $85.3 \%$ (95\% CI 74.6 to 92.8 ) and specificity of $100 \%$ (95\% CI 95.8 to 100). In silico analysis suggest $100 \%$ coverage of available complete genomes for SARS-CoV-2 in TaqPath PCR targeting three genes: N-gene, ORF-1ab and S-gene. ${ }^{34}$

Most guidelines at that time recommended that HCP should be tested between the 12th and 14th day after exposure. ${ }^{14}{ }^{16}$ But this meant that our HCP were separated from their family for a whole 3 weeks, which was unacceptable to them and organising logistic support difficult for the HCP welfare unit. We undertook a literature search to understand transmission in a healthcare setting. We found that if there is no breach in protocol during COVID-19 duty, the pretest probability of getting infected is as low as $<2 \%{ }^{3}$ Also, the median incubation time of SARS-Cov 2 virus was 5.1 days ( $95 \%$ CI 4.5 to 5.8 days) and $97.5 \%$ of those will develop symptoms within 11.5 days. $^{2}$ For a conservative estimate of sensitivity of $75 \%$ and specificity was $95 \%$, we calculated the positive likelihood ratio as 15 (95\% CI 6.34 to 36 ) and a negative likelihood ratio as 0.26 (95\% CI 0.19 to 0.37$).{ }^{35}$ We found that if pretest probability is conservatively taken at even $5 \%$ for those without any documented breach, a single negative test on day 7 , the post-test probability would be $<1 \%$ (95\% CI $0 \%$ to $4 \%$ ) calculated using an online tool. ${ }^{36}$ Thus a negative test, virtually minimised the probability of carrying infection home. This allayed their fear of inadvertently taking the infection to their homes and community. However, we asked them to selfmonitor for symptoms and report back if they had any. So, we could safely return them back to their homes after 7-day postduty isolation instead of recommended 14 days. Thus, our HCP worked for 7 days, rested for 7 days and were tested on the seventh day postduty, after which they could return to their non-COVID duty, if found negative. This helped in optimal utilisation of manpower and early return of HCP to their home. We did pooled sample testing of postduty HCP tests to optimise utilisation of limited COVID-19 testing resources.

\section{HCP empowerment}

We used the HCP's smart phone platform to communicate with them as well as provide all required information/ services. This resulted in a system which prevented fomite transmission and made all the communication paperless and efficient. HCP were also thus empowered to access information, training modules, communicate with the supervisory unit and coworkers, coordinate logistics and recreation using their personal mobile devices (figure 4). The HCP structured feedback questionnaire was also provided on their smart phone platform to understand their needs and views related to background, training, their amenities, physical/psychological support received and problems faced during the complete period of duty and postduty isolation period (see online supplemental file 1). A new website portal was created where we regularly uploaded the information regarding COVID-19 for the HCP. ${ }^{10}$ This COVID-19 portal also had a dashboard which updated the hospital statistics and cumulative data on COVID-19 on a daily basis.

\section{Statistical methods}

Data were presented in the form of appropriate graphs and tables and statistical analyses would be performed using the Microsoft Excel software. Discrete categorical data were presented as $\mathrm{n}(\%)$; continuous data were presented as mean $\pm \mathrm{SD}$ and range or median and IQR, as appropriate.

\section{Patient and public involvement in research}

This protocol deals with the creation of a novel hospital MDT and an HCP-centric policy while tackling the COVID-19 pandemic in our institution. Since this largely concerns HCP, and the process of creating a sustainable and willing healthcare team in a public health setting, the patient and public involvement is indirect as they were beneficiaries of this COVID-19 care model. 


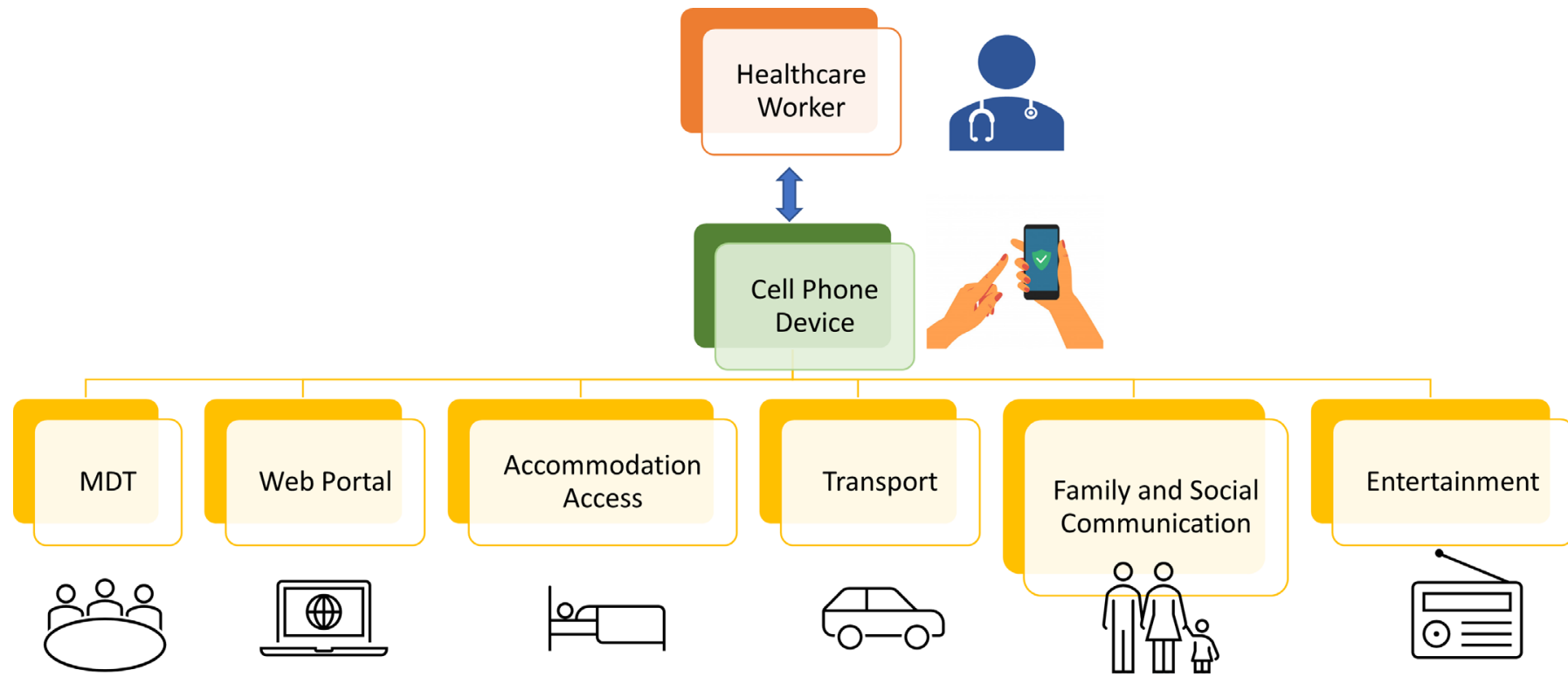

Figure 4 Empowerment of healthcare personnel (HCP). We empowered the HCP by providing all the contact helplines/ services/web portal access/entertainment/standard operating protocols for personal and patient care on their mobile platform to keep all communication efficient and fomite free. MDT, multidisciplinary team.

\section{RESULTS}

Between 31 March and 20 July 2020, we clinically screened 5553 outpatients, of whom 3012 (54.2\%) were COVID-19 suspects, which constituted the medium-risk 'orange zone' of the hospital protocol. The length of stay in the orange zone of COVID-19 care was 8-12 hours. Of all 3012 COVID-19 suspect cases, 346 (11.4\%) tested COVID-19 positive with $57.2 \%$ male preponderance. These patients were shifted to the 'COVID-19 care facility' or 'red zone' which had the highest risk of exposure. The average length of stay was 18.3 days with a recovery rate of $74.6 \%$. There were 19 deaths with mortality rate of $5.4 \%$.

\section{HCP recruitment}

Between 31 March and 20 July 2020, a total of 1224 HCP were trained and recruited for red zone duty including $312(25.4 \%)$ doctors, $382(31.2 \%)$ nurses, $76(6.2 \%)$ technicians, 452 (36.9\%) hospital assistants and 2 physiotherapists $(0.2 \%)$. They were all aged $<50$ years ( $62.4 \%$ male). Sixteen (1.3\%) persons had done 2 tours of duty. Similar protocols were used for the $960 \mathrm{HCP}$ in the orange zone. Around $16 \mathrm{HCP}$ were deployed sequentially in the orange and then the red zones as they were specialised critical care, anaesthesia, internist or dialysis unit HCP. There was a gap of at least 4 weeks between these postings. Eligible HCP were recruited from different departments and after orientation and training, they were deployed in the COVID-19 facility by rotation. The duty period of the frontline team was set up for 7 days in a 6 -hour shift per day. This was followed by self-isolation for a period of 7 days with testing on day 5 or 7 of the last day of exposure. This limited the duration of exposure to patients with COVID-19 and was tolerable in terms of wearing heavy PPE in warm weather conditions. This policy found wide acceptability among the HCP. All $12600 \mathrm{HCP}$ in the PGIMER were trained depending on their area of deployment and followed PPE instructions specific to their area of deployment. The PGIMER PPE guidelines are summarised in online supplemental table 1 .

\section{HCP exposure}

The red zone had full PPE provision, with N95 respirators, coverall suits, visor, cap, gloves and dual shoe covers. Most HCP (94.1\%) had direct contact with the patients with COVID-19 with $87.2 \%$ patients working within $1 \mathrm{~m}$ distance. Aerosol generating procedures such as tracheal intubation, non-invasive ventilation, tracheostomy, cardiopulmonary resuscitation, manual ventilation, gastroduodenoscopy or bronchoscopy were performed by $71.2 \%$ HCP. The exposure duration per patient was $<5 \mathrm{~min}$ per patient in $46.8 \%$ and between 5 and $15 \mathrm{~min}$ in $22.2 \%$ encounters. We performed three gastroduodenoscopies, one orthopaedic surgery, one pericardial drain placement, one pancreatic necrosis drainage and two thoracic drains in the COVID-19 facility without any documented breach during these procedures and no documented transmission.

The length of contact with the patient in orange zone of COVID-19 care was mostly $<15$ min and only a small fraction of patients were positive. Thus, initially only surgical masks and gowns were given here, but this policy was modified to full PPE with N95 respirators with infection control team safety feedback. Full PPE cover was extended to the orange zone subsequently after a period of 2 weeks as two HCP were infected early in this area and increasing the level of PPE was a felt need (figure 4).

\section{HCPs with COVID-19 infection}

None of the HCP $(n=1224)$ documented a breach in PPE in the red zone. One $(0.08 \%)$ of the 1224 HCP in high-risk 
zone tested positive postduty. All such persons underwent contact tracing by the Community Medicine and Infection Control Units. We report there was no transmission in the red zone when HCP were using N95 respirator appropriately and impervious PPE. None of the HCP who were tested negative, subsequently became symptomatic for any COVID-19-like illness, suggesting this protocol of testing was effective.

However, in the orange zone with 960 personnel, 6 $(0.62 \%)$ HCP tested positive after providing patient care to unsuspected patients tested positive for COVID-19 in the first month of pandemic. They reported major breaches in protocol including improper compliance with PPE protocols. Of these six HCP, only two persons were symptomatic with mild cough and all six have since recovered. After institution of re-training and full PPE (N95 and impervious gowns) to this zone we have not documented further transmission. By this time, we had also created location-specific training videos, interactive modules, installed the CCTV system and procured appropriate tried and tested protective gear, which resulted in no more inadvertent risk from hospital exposure.

In the green zone with 12600 employees, a total of 23 $(0.18 \%)$ HCP $(56.5 \%$ female) tested positive for COVID19 , all of whom had definite evidence of community transmission. Of them $14(60.8 \%)$ were asymptomatic, $8(34.7 \%)$ had mild symptoms of fever, sore throat and one $(4.3 \%)$ required low-dose oxygen therapy. Source of exposure and contact tracing was done for all 23 persons. They acquired it while not following social distancing and universal masking norms, when they either attended mass gatherings, social events, had tea or lunch breaks with their office colleagues who later tested positive. So, the overall infection rate for our centre is $30 / 12600$, which is $(0.23 \%)$ including all cases of hospital and community transmission.

\section{Medical and mental healthcare of the HCP}

The members of the HCP welfare unit periodically assessed physical and mental health status by teleconsultations. In the feedback from $122 \mathrm{HCP}, 13$ (23.6\%) had sore throat, $3(5.5 \%)$ had diarrhoea and $3(5.5 \%)$ reported fever. Isolated cases of rhinitis, headache and myalgia were reported. None among them tested positive for COVID-19 and were treated symptomatically for their non-COVID illness.

HCPs were evaluated by the mental healthcare team $(\mathrm{n}=194)$ during the postduty isolation period by a semistructured interview. Of these only, $8.76 \%(n=17)$ were considered to have a clinically diagnosable anxiety disorder and/or mild depressive episode. Other mental health issues identified were insomnia $(\mathrm{n}=17 ; 8.76 \%)$, feeling lonely $(n=46 ; 23.7 \%)$, feeling bored $(n=37$; $19.07 \%)$, feeling irritable $(\mathrm{n}=10 ; 5.15 \%)$, feeling demoralised $(n=13 ; 6.70 \%)$, being afraid of contracting the infection $(\mathrm{n}=57 ; 29.38 \%)$ and feeling scared of infecting family members $(\mathrm{n}=60 ; 30.9 \%)$. Relaxation exercises were advised to the majority of HCP having mental health issues. Psychological support was provided to all those experiencing negative emotional states (feeling lonely, feeling irritable, feeling demoralised, feeling scared and anxious, feeling socially disconnected feeling like running away from work and so on). ${ }^{37} 38$ However, medications (mainly anti-anxiety, benzodiazepines) were prescribed in seven HCP for short duration and in only one HCP, antidepressants had to be initiated. A meditation and yoga/exercise programme were initiated for those who volunteered, during the social isolation period to help maintain the physical and mental health of HCP. ${ }^{39} 40$

\section{Feedback and HCP satisfaction}

We analysed the collected feedback from initially deployed HCP ( $n=122)$ after the COVID-19 posting in the dedicated COVID-19 hospital. All responses were graded on a 5-point Likert scale, with the best result being 5 . We report that $92(75.4 \%)$ HCP agreed that the training received was $\geq 4$ points. About $93(76.2 \%)$ HCP graded $\geq 4$ points about the adequacy of the PPE provided to them. Eighty-seven (71.3\%) scored the communication devices and $95(77.8 \%)$ scored the measures to protect them in the doffing area $\geq 4$ points. The low HCP infection rate of $0.08 \%$ was reassuring. About $92.8 \%$ of HCP were mentally prepared for the duty before entering the COVID-19 wing and $81 \%$ found the psychosocial support adequate during their isolation period. Although most agreed that posting in the COVID-19 area was acceptable, and they will serve again if required, and $54.8 \%$ said they would even volunteer for the duty (online supplemental figure 1). Their only residual concern was that they missed their families during the period of isolation. Overall, all HCP expressed satisfaction with their experience and changed their outlook from trepidation to confidence as they appreciated the HCP centric policy of our institute, which complimented the quality of patient care.

\section{DISCUSSION}

We found that a multidimensional healthcare centric policy developed by our MDT resulted in reduced transmission of COVID-19 among those taking care of patients infected with COVID-19. The HCP-centric policy of limiting work shifts of frontline workers to 6 hours ensured rest and recreation, reduced cumulative exposure to COVID-19 patients and the probability of HCP infection. All our 30 infected HCP had no/mild symptoms and recovered completely and overall HCP infection rate was $0.23 \%$. Only one infection was documented in the red zone. She was a respiratory physiotherapist who did not report any breach in PPE while taking care of patients with COVID-19 in the red zone. She possibly acquired it from the community while not following social distancing and universal masking policy just prior to her COVID-19 duty shift. As she remained asymptomatic and was tested as per protocol only at the seventh day postduty, this remained undetected throughout her entire duty period. Her contacts were traced and all tested 
negative. Community transmission was considered to be the source of COVID-19 infection for an HCP if the HCP always was in the prescribed PPE according to the site of duty, there was no breach and there was instead high-risk exposure to a COVID-19-positive/COVID-19 suspect in the community. The HCP would be interviewed by the Contact Tracing team (Community Medicine and Infection Control units). The possible sources of acquiring COVID-19 was ascertained by a detailed assessment of their at-risk activities in the preceding 2 weeks.

Our experience contrasted with the global experience, where many HCP were infected with COVID-19. The National Health Commission of the People's Republic of China reported that up to 24 February 2020, 2055 HCP were confirmed to be infected with COVID-19, $90 \%$ of whom were from Hubei province, with $22(1.1 \%)$ deaths, but these events were not defined as being hospitalacquired or community-acquired infection. ${ }^{11}$ Among these reported deaths of HCP, Dr Li Wenliang, the ophthalmic surgeon who first raised the alarm about a novel viral pneumonia, became the face of the threat to HCP in the global pandemic. ${ }^{41}$ Our model differed from reports from other countries ${ }^{42-45}$ as we ensured that our HCP had adequate PPE, mandatory use of N95 respirators and impervious gowns in the COVID-19 facility, duration of shift in PPE was just 6 hours, making the HCP comfortable, and ensuring meals and washroom usage on time. We also provided transport, boarding and lodging for those who needed it, we offered end of COVID-19 posting voluntary testing to all HCP who requested it and mandatory testing for those who were symptomatic, highrisk contacts or reported a breach in PPE. Our population and our HCP tend to be of a younger demographic and tend not to have very severe disease.

The data from Italy are particularly worrisome as over $12680 \mathrm{HCP}$ were infected, with almost 100 physicians and 26 nurses losing their lives to the COVID-19 effort as of 7 April 2020. ${ }^{42} 44$ Another case study is that of Spain, where the rate of infection of HCP is reported to be about $20 \%$ of the total COVID-19 cases, mainly due to lack of adequate PPE. As of 1 June, about 50000 HCP were reported infected, many of whom were critically ill and about 70 had died. ${ }^{43}$ In the USA, the CDC documented 9282 COVID-19 cases in HCP, of whom 723 $(8 \%-10 \%)$ were hospitalised and $184(2 \%-5 \%)$ were admitted to intensive care units. ${ }^{44}$ Reports from Brazil suggest high HCP infections. ${ }^{45}$ Currently, WHO estimates there are 13.8 million COVID-19 infections worldwide and that HCP represent 1.4 million cases, about $10 \%$ of all COVID-19 cases. ${ }^{46}$ This sobering thought suggests that even in resource-poor setting like ours, if we are ready to make suitable HCP-centric policy, we may save our frontline healthcare workers. We were ready to adapt and innovate, which helped us in ensuring HCP safety and welfare in trying and uncertain times.

We realised that welfare needs of HCP are dynamic and needed to be individualised. We had an enormous task segregating the three tiers of COVID-19 care areas, training and protecting HCP and ensuring adequate manpower at screening, testing and patient care sites, while maintaining the usual academic and non-COVID-19 patient care work schedule of a tertiary care university hospital. ${ }^{29}$

India has had a delay in peak in cases due to imposition of an early countrywide lockdown, which opened up in a phased manner from 1 June 2020. We used this time effectively to prepare for the COVID-19 effort and creation of dedicated laboratories, COVID-19 care facilities and screening and quarantine centres. At our centre, the risk of COVID-19 infection to the HCP was $<1 \%$ overall, which is much lower than global figures. ${ }^{156}$

Professionally supervising the physical and mental health of our HCP while they are fighting the CORONA war was a challenge for us. Our HCP were pressed into duty in trying circumstances, were anxious and had genuine concerns. ${ }^{20} 40$ This HCP welfare model was created in a dynamic manner, in consultation with the MDT, and in an iterative manner taking all relevant feedback from HCP into consideration. An adaptive model is likely to survive the challenge in the long-haul fight against COVID-19 yielding high HCP satisfaction and effective patient care.

Acquiring initial feedback from the first responder HCP and implementing reforms was an integral part of the welfare team. The feedback was collected from 122 initial consecutive HCP including residents, nurses, technicians and sanitation workers. This vital information was assessed in April itself and allowed us to improve lodging access, streamline the online access forms, prepare palatable food menus and organise timely transport to subsequent HCP have continued to provide unstructured feedback through our established communication channel. The profile of the initial 122 individuals and subsequent HCP is similar in terms of age, gender and cadre. The data are thus representative of all the HCP, in the COVID-19 care facility. Most (57\%) HCP expressed willingness to repeat their COVID-19 duty given their positive experience. A dynamic interaction between MDT, HCP and other stakeholders lead to sustainable model with high degree of employee satisfaction.

The COVID-19 welfare unit remedied most of the concerns of the HCP by providing a comprehensive health, adequate PPE, lodging, transport and timely financial support package, which helped in maintaining their morale and overall well-being resulted in HCP passing on the positive feedback to subsequent batches resulting in a willing renewable workforce. ${ }^{22}$

Our hospital setting is representative of a tertiary care university hospital, one of several public and private institutes in India which have been designated as COVID-19 care centres. However, there is no published health policy-related data from these centres which we can readily compare. The government has designated a threetier system for COVID-19 care: asymptomatic and mildly symptomatic are to be isolated in COVID-19 care centres, significantly symptomatic cases in equipped hospitals and complicated COVID-19 cases at tertiary care apex 
institutions. Thus, our hospital had more serious symptomatic cases, patients requiring invasive ventilation, comorbidities and need for interventions increasing our HCP exposure rates and requirement of intensive care. In the initial phase of the pandemic, in the months of March through June, every single case of COVID-19, asymptomatic or otherwise, was admitted with the objective of preventing community spread. Private hospitals in the country were permitted to admit patients under strict regulation by the state governments, fee caps and a strict protocol for admitting or referring cases. Community healthcare was provided at the level of the primary health centres with 'anganwadi' and accredited social health activist workers as frontline workers in both urban and rural areas. Secondary level care was provided in large district hospitals and civil hospitals which were designated as COVID-19 care facilities for symptomatic patients. Nodal centres, such as our institute, supervised many such smaller units and supported their core teams of HCP. In addition, our team has provided training and scaled-down Treatment Unit Models for secondary level care at numerous centres in North India. Using web-based and mobile-based model for communication ensure format-free efficient low-cost communication. Also, the telemedicine model for routine monitoring reduced exposure of our HCP.

PGIMER honoured the services provided by the HCP in the COVID-19 frontline by awarding a certificate of appreciation to our 'CORONA WARRIORS'. Their effort was praised and highlighted in the press and media. This also stimulated our other HCP to come forward for COVID-19 duty. Our model's main limitation is that India is still having a rising COVID-19 curve, with population heterogeneity in different parts of the country requiring the MDT to redouble efforts, manage limited resources, brace for more patients and protect the HCP at the same time. We were unable to get the feedback forms for all HCP which was another limitation. Other limitations include the cultural and social factors unique to India and a variable peak of infection in different cities that results in waves of new cases. Our model was generated in anticipation of the rising curve and we had ample time to prepare the COVID-19 facility, the isolation lodging unit and stockpile adequate good quality PPE. Ours is a large tertiary care university hospital with good infrastructure and expertise, which worked to our advantage. Our population and our HCP tend to be of a younger demographic and tend not to have very severe disease. We did not require additional manpower for the COVID-19 effort and were able to divert human resources from defunctionalised units like routine operating rooms, and outpatient clinics. India has low cost internet access providers, and the use of telemedicine allowed us to maintain routine patient care. Our model also requires good administrative support, and use of mobile internet services, which may not be readily available at other sites. Using web-based and mobile-based model for communication ensured efficient low-cost communication. Also, the telemedicine model for routine monitoring reduced exposure of our HCP. However, scaled down versions can be applied in the local setting as per caseload and HCP needs. We have shared our team's real-life experience of a tertiary care centre in a low-income and middle-income country to ensure a safe working environment for our HCP. By using existing resources, we created a low-cost model for COVID-19 care. In the future, this model can be replicated in setting up COVID-19 hospital services in secondary and tertiary level care institutions in India and elsewhere. ${ }^{29} 39$

\section{CONCLUSION}

A multidimensional HCP-centric policy was effective in ensuring safety, satisfaction and welfare of HCP in a resource-poor setting and resulted in a willing workforce to fight the pandemic. An MDT is required to create and sustain the model. The model needs to be dynamic, responsive to feedback, iterative and innovative. Ensuring adequate appropriate PPE, and monitoring its use, are essential in reducing patient to HCP transmission. Ample use of low-cost accessible technology made it efficient, effective and paperless, and it possibly reduced fomite transmission. Our overall COVID-19 transmission among $\mathrm{HCP}$ is among the lowest reported yet at $0.23 \%$. This realworld data from India show that training, empowering, support and protecting HCP results in high satisfaction rates, a willing workforce and sustainable use of human resources. This model is reproducible in other low-income and middle-income countries and other resource-poor settings, where the pandemic is still unfolding.

\section{Author affiliations}

${ }^{1}$ Department of Gastroenterology, PGIMER, Chandigarh, India

${ }^{2}$ Department of Hepatology, PGIMER, Chandigarh, India

${ }^{3}$ Department of Anatomy, PGIMER, Chandigarh, India

${ }^{4}$ Department of Psychiatry, PGIMER, Chandigarh, India

${ }^{5}$ Department of Community Medicine and School of Public Health, PGIMER, Chandigarh, India

${ }^{6}$ Department of Hospital Administration, PGIMER, Chandigarh, India

${ }^{7}$ Department of Microbiology, PGIMER, Chandigarh, India

${ }^{8}$ Department of Internal Medicine, PGIMER, Chandigarh, India

${ }^{9}$ Department of Virology, PGIMER, Chandigarh, India

${ }^{10}$ Department of Anesthesia, PGIMER, Chandigarh, India

${ }^{11}$ Department of Neurosurgery, PGIMER, Chandigarh, India

${ }^{12}$ Department of Pediatrics, PGIMER, Chandigarh, India

${ }^{13}$ Department of Dietetics, PGIMER, Chandigarh, India

${ }^{14}$ Department of Parasitology, PGIMER, Chandigarh, India

${ }^{15}$ Department of Pulmonology, PGIMER, Chandigarh, India

${ }^{16}$ Department of Ophthalmology, PGIMER, Chandigarh, India

Acknowledgements The authors would like to thank Mr Vikas Yadav and Mr Sonu Verma for helping with secretarial work for the team, Mr Narinder Kumar Prarthi for helping in transport and all PGIMER staff for assisting in the management of the COVID-19 pandemic.

Contributors UD is the principal author, and developed the study concept, drafted the policy and manuscript and is the convenor of the HCP Welfare Committee. AS and MP drafted the manuscript. TG was responsible for transport and accommodation. SSa was involved in training. SG was the primary mental health expert, and all the principal coauthors helped administer the HCP policy. SSh, PVML, ST, MB, VS, BG, RC, BB, JS, PA, RM, SM all contributed to the HCP welfare team, and aided data collection. MPS and AG were coauthors from the virology testing 
team. GS, RRG, VK, AK, RSB, AKA, KG, PaM, NY, PrM, JST, RS, ISS, RA, MJ, AB, SJ, RK, AC and GDP were part of the COVID-19 patient care team or administrative unit involved with aspects of patient care and administration. JR is the director, who had intellectual contribution to the policy and was the administrative head of the protocol.

Funding The authors have not declared a specific grant for this research from any funding agency in the public, commercial or not-for-profit sectors.

Competing interests None declared.

Patient consent for publication Not required.

Ethics approval Ethical clearance for reporting the results of our HCP protocol and feedback was taken from the Institutional Ethics Committee (Letter No INT/ IEC/2020/SPL997; Dated 25 July 2020) and the need for individual informed consent was waived. This retrospective study was conducted in accordance with the Declaration of Helsinki and ICMR guidelines. All authors had access to the manuscript data and approved the final draft.

Provenance and peer review Not commissioned; externally peer reviewed. Data availability statement № data are available.

Supplemental material This content has been supplied by the author(s). It has not been vetted by BMJ Publishing Group Limited (BMJ) and may not have been peer-reviewed. Any opinions or recommendations discussed are solely those of the author(s) and are not endorsed by BMJ. BMJ disclaims all liability and responsibility arising from any reliance placed on the content. Where the content includes any translated material, BMJ does not warrant the accuracy and reliability of the translations (including but not limited to local regulations, clinical guidelines, terminology, drug names and drug dosages), and is not responsible for any error and/or omissions arising from translation and adaptation or otherwise.

Open access This is an open access article distributed in accordance with the Creative Commons Attribution Non Commercial (CC BY-NC 4.0) license, which permits others to distribute, remix, adapt, build upon this work non-commercially, and license their derivative works on different terms, provided the original work is properly cited, appropriate credit is given, any changes made indicated, and the use is non-commercial. See: http://creativecommons.org/licenses/by-nc/4.0/.

\section{ORCID iDs}

Madhumita Premkumar http://orcid.org/0000-0003-2961-4148

Sugandhi Sharma http://orcid.org/0000-0001-7828-3547

J S Thakur http://orcid.org/0000-0003-1339-1429

Inderpaul Singh Sehgal http://orcid.org/0000-0002-6505-6019

Rakesh Kochhar http://orcid.org/0000-0002-4077-6474

\section{REFERENCES}

1 Wu Z, McGoogan JM. Characteristics of and important lessons from the coronavirus disease 2019 (COVID-19) outbreak in china: summary of a report of 72314 cases from the chinese center for disease control and prevention. JAMA 2020;323:1239-42.

2 Guan W-J, Ni Z-Y, Hu Y, W-j G, Z-y N, et al. Clinical characteristics of coronavirus disease 2019 in China. N Engl J Med 2020;382:1708-20.

3 Sohrabi C, Alsafi Z, O'Neill N, O'Neill N, et al. World Health organization declares global emergency: a review of the 2019 novel coronavirus (COVID-19). Int J Surg 2020;76:71-6.

4 WHO. WHO Dashboard, 2020. Available: https://www.who.int/ redirect-pages/page/novel-coronavirus-(COVID-19)-situationdashboard

5 Government of India. COVID-19 statewise status, 2020. Available: https://www.mygov.in/COVID-19

6 Chatterjee P, Anand T, Singh KJ, et al. Healthcare workers \& SARSCoV-2 infection in India: A case-control investigation in the time of COVID-19. Indian J Med Res 2020;151:459-67.

7 Science the wire. IMA slam centre, publishes list of 382 doctors who died due to COVID -19, 2020. Available: https://science.thewire.in/ health/ima-health-minister-harsh-vardhan-health-workers-covid-19deaths/

8 Bagcchi S. Stigma during the COVID-19 pandemic. Lancet Infect Dis 2020;20:782

9 Bhaumik S, Moola S, Tyagi J, et al. Community health workers for pandemic response: a rapid evidence synthesis. BMJ Glob Health 2020;5:e002769.

10 pgimer. PGIMER COVID-19 SOP, 2020. Available: http://pgimer.edu. in/PGIMER_PORTAL/PGIMERPORTAL/covid19/healthcare.html
11 Surging news. Infection of over 3000 medical staff in Hubei, 2020. Available: https://mp.weixin.qq.com/s/mnWPipga4ApDdrAYTrM5qA

12 The Lancet. COVID-19: protecting health-care workers. The Lancet 2020;395:922.

13 Wang J, Zhou M, Liu F. Reasons for healthcare workers becoming infected with novel coronavirus disease 2019 (COVID-19) in China. J Hosp Infect 2020;105:100-1.

14 World Health Organization. Report of the WHO-China joint mission on coronavirus disease 2019 (COVID-19), 2020. Available: https:// www.who.int/docs/default-source/coronaviruse/who-china-jointmission-on-COVID-19-final-report.pdf

15 World Health Organization. Coronavirus disease (Covid-19) outbreak: rights, roles and responsibilities of health workers, including key considerations for occupational safety and health, 2020. Available: https://www.who.int/docs/default-source/ coronaviruse/who-rights-roles-respon-hw-covid-19.pdf?sfvrsn= bcabd401 0

16 Centers for Disease Control and Prevention (CDC). About coronavirus disease 2019 (COVID-19), 2020. Available: https://www. cdc.gov/coronavirus/2019-ncov/about/

17 Ministry of Health and Family Welfare Directorate General of Health Services (EMR Division), Government of India. Advisory for managing health care workers working in COVID and Non-COVID areas of the hospital dated 18th June 2020, 2020. Available: https://www.mohfw. gov.in/pdf/updatedAdvisoryformanaging Healthcareworkersworkingin COVIDandNonCOVIDareasofthehospital.pdf

18 Ministry of Health and Family Welfare Directorate General of Health Services (EMR Division), Government of India. Rational use of personal protective equipment, 2020. Available: https://www.mohfw. gov.in/pdf/GuidelinesonrationaluseofPersonalProtectiveEquipment. pdf

19 Ministry of Health and Family Welfare Directorate General of Health Services (EMR Division), Government of India. Advisory for human resource management of COVID-19, 2020. Available: https://www. mohfw.gov.in/pdf/AdvisoryforHRmanagement.pdf

20 Lai J, Ma S, Wang Y, et al. Factors associated with mental health outcomes among health care workers exposed to coronavirus disease 2019. JAMA Netw Open 2020;3:e203976.

21 Ağalar C, Öztürk Engin D. Protective measures for COVID-19 for healthcare providers and laboratory personnel. Turk J Med Sci 2020;50:578-84.

22 Chersich MF, Gray G, Fairlie L, et al. COVID-19 in Africa: care and protection for frontline healthcare workers. Global Health 2020;16:46

23 Liu M, Cheng S-Z, Xu K-W, et al. Use of personal protective equipment against coronavirus disease 2019 by healthcare professionals in Wuhan, China: cross sectional study. BMJ 2020;369:m2195.

24 Pappa S, Ntella V, Giannakas T, et al. Prevalence of depression, anxiety, and insomnia among healthcare workers during the COVID-19 pandemic: a systematic review and meta-analysis. Brain Behav Immun 2020;88:901-7.

25 Grover S, Dua D, Sahoo S, et al. Why all COVID-19 hospitals should have mental health professionals: the importance of mental health in a worldwide crisis! Asian J Psychiatr 2020;51:102147.

26 Ferioli M, Cisternino C, Leo V, et al. Protecting healthcare workers from SARS-CoV-2 infection: practical indications. Eur Respir Rev 2020;29:200068.

27 Sharma R, Saini NK. A critical appraisal of Kuppuswamy's socioeconomic status scale in the present scenario. J Family Med Prim Care 2014;3:3-4.

28 World Health Organization. Health workers: a global profile, 2020 Available: https://www.who.int/whr/2006/06_chap1_en.pdf

29 Pandey N, Kaushal V, Puri GD, et al. Transforming a general Hospital to an infectious disease Hospital for COVID-19 over 2 weeks. Front Public Health 2020;8:382.

30 National Centre for Disease Control. Covid 19 outbreak. Guidelines for setting up an isolation facility or ward, 2020. Available: https:// ncdc.gov.in/WriteReadData/l892s/42417646181584529159.pdf

31 Ministry of Health and Family Welfare, Government of India. National guidelines for infection prevention and control in healthcare available from facilities, 2020. Available: https://www.mohfw.gov.in/pdf/ National\%20Guidelines\%20for\%20IPC\%20in\%20HCF\%20-\% 20final\%281\%29.pdf

32 Xiang Y-T, Yang Y, Li W, et al. Timely mental health care for the 2019 novel coronavirus outbreak is urgently needed. Lancet Psychiatry 2020;7:228-9.

33 Kuzman MR, Curkovic M, Wasserman D. Principles of mental health care during the COVID-19 pandemic. Eur Psychiatry 2020;63:e45.

34 Matsumura Y, Shimizu T, Noguchi T, et al. Comparison of 12 molecular detection assays for severe acute respiratory syndrome coronavirus 2 (SARS-CoV-2). J Mol Diagn 2021;23:164-70. 
35 Lauer SA, Grantz KH, Bi Q, et al. The incubation period of coronavirus disease 2019 (COVID-19) from publicly reported confirmed cases: estimation and application. Ann Intern Med 2020;172:577-82.

36 Schwartz A. Diagnostic test calculator (version 2010042101). Copyright (c) 2002-2006 (Copyright (c) 2002-2007 by Alan Schwartz alansz@uic.edu), 2006. Available: http://araw.mede.uic.edu/cgialansz/testcalc.pl

37 Mehra A, Sahoo S, Verma M. Psychological issues faced by the health care workers during the COVID-19 pandemic. J Postgrad Med Educ Res 2020;54:88-93.

38 Pfefferbaum B, North CS. Mental health and the Covid-19 pandemic. N Engl J Med 2020;383:510-2.

39 Christopher DJ, Isaac BT, Rupali P, et al. Health-care preparedness and health-care worker protection in COVID-19 pandemic. Lung India 2020;37:238-45.

40 Sahoo S, Singh G, Bhogal RPS. Psychosocial issues among the "faceless corona warriors" - the hospital housekeeping staff and sanitary workers on COVID-19 duty: an exploratory survey from a tertiary health care center from North India. J Postgrad Med Educ Res 2020;54:94-9.
41 Petersen E, Hui D, Hamer DH, et al. Li Wenliang, a face to the frontline healthcare worker. The first doctor to notify the emergence of the SARS-CoV-2, (COVID-19), outbreak. Int $J$ Infect Dis 2020;93:205-7.

42 Barranco R, Ventura F. Covid-19 and infection in health-care workers: an emerging problem. Med Leg J 2020;88:65-6.

43 Crespo J, Calleja JL, Zapatero A. Widespread covid-19 infection among Spanish healthcare professionals did not occur by chance, 2020. Available: https://blogs.bmj.com/bmj/2020/06/01/widespreadcovid-19-infection-among-spanish-healthcare-professionals-did-notoccur-by-chance/

44 Characteristics of health care personnel with COVID-19 - United States February 12-April 9, 2020. MMWR Morb Mortal Wkly Rep;2020:477-81.

45 Pessa Valente E, Cruz Vaz da Costa Damásio L, Luz LS, et al. COVID-19 among health workers in Brazil: the silent wave. J Glob Health 2020;10:010379.

46 World Health Organization. WHO Director-General's opening remarks at the media briefing on COVID-19-17 July 2020, 2020. Available: https://www.who.int/dg/speeches/detail/who-director-general-sopening-remarks-at-the-media-briefing-on-covid-19---17-july-2020 